Review

For reprint orders, please contact: reprints@futuremedicine.com

\title{
Translating extracellular microRNA into clinical biomarkers for drug-induced toxicity: from high-throughput profiling to validation
}

Over the past 5 years, extracellular microRNAs (miRNAs) are being vigorously explored as injury biomarkers, including drug-induced cardiotoxicity, hepatotoxicity and nephrotoxicity. Currently, the development of miRNAs as clinical biomarkers has been hindered by the lack of standardization. Therefore, extracellular miRNAbased biomarkers have not been embraced as diagnostic tools. Each platform has its strengths and weaknesses when working with low-input-amount RNA samples from body fluids; the selection of a miRNA quantification approach should be based on the study design. The following review provides a summary of the extracellular miRNA release and stability in body fluids, performances of different miRNA quantification platforms, existing clinical gold standards for drug-induced tissue damage and translation of the miRNA biomarkers from the nonclinical to clinical setting.

Keywords: biomarkers $\bullet$ cardiotoxicity $\bullet$ extracellular $\bullet$ hepatotoxicity $\bullet$ microRNA

- nephrotoxicity

MicroRNAs (miRNAs) are small non-coding RNAs approximately 22 bp in length, that regulate gene expression post-transcriptionally by binding and inhibiting particular mRNA targets. Currently, more than 1800 miRNAs have been identified and it appears that $>60 \%$ of human protein-coding genes are regulated by miRNAs [1]. They typically downregulate gene expression by targeting mRNA degradation or interfering with protein synthesis process via base-pairing with complementary sequences within mRNA [2,3]. In the early 1990s, the first miRNA, lin-4, was identified in Caenorhabditis elegans [4]. However, it was not until 10 years later that miRNAs were recognized as unique biological regulators. During the past decade, we have learned much about the basic mechanisms of miRNA biogenesis and involvement in normal cellular function. However, more recently it has become apparent that dysregulation of miRNA has been associated with human disease. In 2008, novel reports demonstrating the presence of miRNAs in body fluids were published [5,6], since then a large number of studies have been conducted to explore miRNAs as sensitive and specific biomarkers for disease and toxicity. Several publicly available databases, miR2Disease [7] and the Human microRNA Disease Database (HMDD) [8], have been created and are valuable resources for investigating the association between miRNA and human disease.

It has been reported that miRNAs are expressed in all mammalian cells and are well conserved among species, ranging from worms to humans. In animals, miRNA have been shown to play essential roles in cellular activities, such as development, cellular differentiation, proliferation, apoptosis, cellcycle control, metabolism and cancer. Similar to mRNA, some miRNA expression is controlled in cell- or tissue-specific manners. For instance, miR-122 is one of the dominate miRNAs in hepatocytes and variants of miR-122 accounted for approximately $72 \%$ of the total liver miRNA population [9]. To gain insights into tissue-specific miRNA distribution, we used small RNA sequencing to
Wenjun Wang ${ }^{1,2}$, Qiang Shi², Williams B Mattes², Donna L Mendrick ${ }^{2} \&$ Xi Yang ${ }^{\star, 2}$

${ }^{1}$ College of Life Science, South-Central University for Nationalities, Wuhan 430074, PR China 2Division of Systems Biology, National Center for Toxicological Research, Food \& Drug Administration, 3900 NCTR Road, Jefferson, AR 72079, USA

*Author for correspondence:

Tel.: +1 8705437321

Fax: +1 8705437736

Xi.Yang@fda.hhs.gov 


\section{(A) Heart-enriched miRNA}

\begin{tabular}{|c|c|c|c|c|c|c|c|c|c|c|}
\hline miRNA & 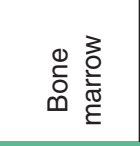 & $\frac{\sqrt{\pi}}{\frac{\pi}{\omega}}$ & 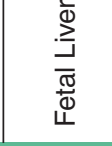 & 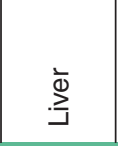 & 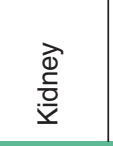 & $\stackrel{\text { D }}{\Xi}$ & 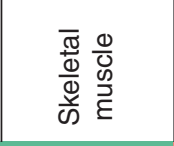 & 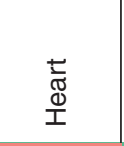 & $\begin{array}{l}\frac{5}{0} \\
\frac{\mathbb{0}}{0} \\
\text { ஸे }\end{array}$ & $\frac{0}{\frac{0}{0}}$ \\
\hline hsa-miR-197-5p & 0 & 0 & 0 & 0 & 0 & 0 & 0 & 190.476 & 0 & 0 \\
\hline hsa-miR-23b-5p & 0 & 0 & 0 & 0 & 0 & 0 & 0 & 190.476 & 0 & 0 \\
\hline hsa-miR-4461 & 0 & 0 & 0 & 0 & 0 & 0 & 0 & 190.476 & 0 & 0 \\
\hline hsa-miR-490-3p & 0 & 569.564 & 291.773 & 174.612 & 0 & 545.455 & 0 & 67476.2 & 0 & 0 \\
\hline hsa-miR-1287 & 0 & 91.5882 & 51.9991 & 69.8446 & 94.4287 & 227.273 & 0 & 1380.95 & 0 & 160 \\
\hline hsa-miR-1 & 218.1818 & 120.21 & 0 & 69.8446 & 0 & 0 & 8000 & 1285.71 & 0 & 0 \\
\hline hsa-miR-133a & 1672.7273 & 1906.18 & 167.553 & 1004.02 & 2696.46 & 15136.4 & 19477583.33 & 2861524 & 4200 & 82000 \\
\hline hsa-miR-208b & 0 & 37.2077 & 0 & 0 & 0 & 0 & 500 & 3190.48 & 0 & 0 \\
\hline hsa-miR-499a-5p & 0 & 297.662 & 43.3326 & 261.917 & 0 & 0 & 916.6667 & 1190.48 & 0 & 0 \\
\hline hsa-miR-378g & 0 & 0 & 0 & 52.3834 & 0 & 0 & 833.3333 & 1000 & 0 & 0 \\
\hline
\end{tabular}

\section{(B) Liver-enriched miRNA}

\begin{tabular}{|c|c|c|c|c|c|c|c|c|c|c|}
\hline miRNA & 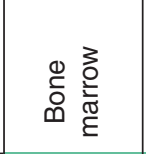 & $\frac{\sqrt[5]{\pi}}{\bar{\oplus}}$ & 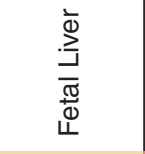 & $\stackrel{\bar{D}}{\stackrel{亠}{J}}$ & 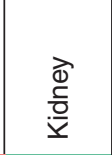 & 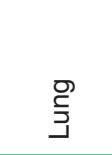 & 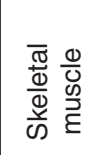 & 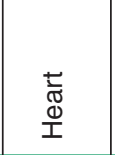 & $\begin{array}{l}\frac{5}{\Phi} \\
\frac{0}{0} \\
\text { की }\end{array}$ & 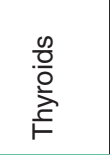 \\
\hline hsa-miR-12 & 0.00 & 0.00 & 14.44 & 200. & 0.0 & $0 . C$ & 0.00 & 0.0 & 0.00 & 0.00 \\
\hline hsa-m & 0 & 20.03 & & & & & 0.00 & 0.0 & & 0.0 \\
\hline hsa-miR-122-3p & 0.00 & 0.00 & 1773.75 & 742 & 0.00 & 0.00 & 0.00 & 0.00 & 0.00 & 0.00 \\
\hline hsa-miR-122-5p & 890.91 & 123.071 & 1818367.23 & 5948620.57 & 839.37 & 1272.73 & 5083.33 & 3000.00 & 400.00 & 1720.00 \\
\hline hsa-miR-548b-5p & 0.00 & 0.00 & 0.00 & 165.88 & 0.00 & 0.00 & 0.00 & 0.00 & 0.00 & 0.00 \\
\hline hsa-miR-4662a-5p & 0.00 & 131.66 & 323.55 & 2470.75 & 41.97 & 0.00 & 0.00 & 0.00 & 0.00 & 0.00 \\
\hline hsa-miR-148a-3p & 419327.27 & 7919.52 & 316252.60 & 2222734.42 & 9579.27 & 24681.82 & 3750.00 & 8047.62 & 10000.00 & 20880.00 \\
\hline hsa-mi & 0.00 & 0.00 & 268.66 & 1981.84 & 83.94 & 0.00 & 0.00 & 0.00 & 0.00 & 0.00 \\
\hline hsa-mi & 0.00 & 2398.47 & 1655.30 & 13253.01 & 0.00 & 0.00 & 3000.00 & 0.00 & 0.00 & 160.00 \\
\hline hsa-miR-148a-5p & 4563.64 & 108.76 & 4289.92 & 22359.00 & 723.95 & 1500.00 & 0.00 & 238.10 & 466.67 & 1280.00 \\
\hline
\end{tabular}

\section{(C) Kidney-enriched miRNA}

\begin{tabular}{|c|c|c|c|c|c|c|c|c|c|c|}
\hline miRNA & 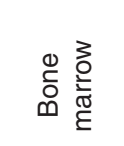 & 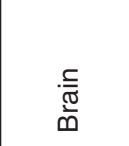 & 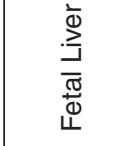 & 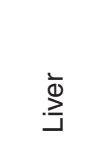 & $\begin{array}{l}\frac{\vec{d}}{\frac{c}{0}} \\
\frac{0}{y}\end{array}$ & $\stackrel{\text { g) }}{\Xi}$ & 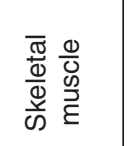 & $\begin{array}{l}\frac{t}{\mathbb{J}} \\
\stackrel{\mathbb{I}}{I}\end{array}$ & $\begin{array}{l}\frac{c}{\Phi} \\
\frac{0}{0} \\
\text { के }\end{array}$ & $\begin{array}{l}\frac{0}{0} \\
\stackrel{0}{\frac{0}{2}} \\
\stackrel{1}{F}\end{array}$ \\
\hline hsa-miR-2116-3p & 0 & 0 & 0 & 0 & 62.9525 & 0 & 0 & 0 & 0 & \\
\hline hsa-miR-1269b & 0 & 0 & 0 & & 146.8891 & 0 & 0 & 0 & 0 & 00 \\
\hline hsa-miR-204-3p & 0 & 11.4485 & 0 & & 2507. & 0 & 0 & 0 & 0 & 440 \\
\hline hsa-miR-184 & 0 & 177.4521 & 1120.869 & & 2255.797 & 0 & 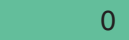 & 0 & 0 & 0 \\
\hline hsa-miR-513a-5p & 0 & 0 & 0 & & 146.8891 & 0 & 0 & 0 & 0 & 0 \\
\hline hsa-miR-30c-2-3p & 127.2727 & 269.0403 & 83.7763 & 759.56 & 10922.25 & 25002 & 666.6667 & 1413 & 333.333 & 2160 \\
\hline hsa-miR-3 & 72.7273 & 88.7261 & 28.8884 & 69.8446 & 283.2861 & 0 & 0 & 0 & 0 & 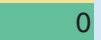 \\
\hline hsa-miR-500a-5p & 72.7273 & 11.4485 & 0 & & 346.2386 & 0 & 0 & 0 & 0 & 160 \\
\hline hsa-miR-30a-3p & 909.0909 & 1863.247 & 447.7698 & 008 & 11331.44 & 4556 & 667 & 48 & 1400 & 2560 \\
\hline hsa-miR-500a-3p & 2010182 & 1416.755 & 4053.039 & 7237646 & 63760.363 & 30409.09 & 305.00 & 43 & 47400 & 19720 \\
\hline
\end{tabular}

Figure 1. Tissue-specific miRNAs quantified by small RNA sequencing. Total RNA from human RNA tissue panel was purchased and microRNAs were quantified by Upper Quartile normalization method using next-generation sequencing. Equal amounts of total RNA were used and the expression levels were presented for each miRNA. In order to present the tissue specificity, a conditional formatting that uses a color scale to differentiate high (red), medium (yellow) and low (green) values was used for each miRNA's expression levels among the tissues. The abundancy of miRNA within the tissue can be compared by their expression levels. 
analyze the miRNA profiles in selected human tissue panel (bone marrow, brain, fetal liver, heart, kidney, liver, lung, skeletal muscle, spleen and thyroids). The top ten miRNA specifically enriched in heart, liver and kidney are listed in Figure 1, with the counts values presented for each miRNA and color coded according to each miRNA's expression levels among the tissues. Similar to previous findings [10,11], miR-122-3p and miR-122-5p levels are highly enriched in the adult liver, with several thousand-fold higher expression compared with other tissues. Some miRNAs are enriched in cardiac tissue and detected at low abundance (miR197-5p, miR-23b-5p and miR-4461); others are detected in both skeletal muscle and heart (miR-1, miR-133a, miR-499, miR-208b and miR-378g). MiR$30 c-2-3 p$ was abundant in the kidney with approximately ten-times higher expression level than other tissues, and miR-2116-3p, miR-1269b and miR-513a-5p showed kidney-specific expression patterns. Ideally, the altered extracellular miRNA after injury should be tissue-specific, such as miR-122. However, a recent study conducted in adults with APAP hepatotoxicity suggested that the highest elevations of circulating miRNAs did not come from liver [12]. One possible reason is that these miRNAs interact with key molecules in cell death and proliferation; therefore, it is not surprising to see their expression in multiple organs. In fact, some of the altered miRNAs (e.g., miR-21) in body fluids have been reported to be associated with various diseases [6].

Drug-induced cardiovascular, liver and renal injuries are the major problems that can result in failures during multiple stages of the drug development process [13]. Adverse drug reactions, as one of the leading causes of morbidity and mortality in healthcare, constitute a major clinical concern. Because of this, the 'omics' technologies have been used to explore translational biomarkers to detect the safety liabilities reliably in animals and human [14]. A biomarker is defined as a 'characteristic that is objectively measured and evaluated as an indicator of normal biological processes, pathogenic processes, or biological responses to a therapeutic intervention' [15,16]. Ideally, novel biomarkers can be identified that will be able to monitor early indications of tissue injury to better inform clinical and regulatory decisions. A good clinical biomarker should have the following characteristics: preferentially produced in target tissue, data can be used to bridge nonclinical and clinical species, display early change for therapeutic intervention, detected through robust analytical assays and easily accessed through body fluids such as blood or urine.

In this article, we provide an overview of current knowledge of extracellular miRNA biomarkers focus- ing on their turnover, stability and forms of release in body fluids. Next, the technical challenges and clinical potential of extracellular miRNAs will be discussed. At last, a review of the nonclinical and clinical findings for using extracellular miRNAs as biomarkers of druginduced cardiotoxicity, hepatotoxicity and nephrotoxicity. Certain properties of miRNAs are considered to be critical for their clinical potential: tissue origin of the biomarker and the release mechanism should be considered and clarified; sensitive to early injury in order to outperform the traditional biomarkers; specificity and correlation with established outcome measures; baseline and interindividual variance should be characterized in supportive clinical studies. Finally, reliable, quick and cost-effective extracellular miRNA quantification assays are required to promote the translation of animal miRNA biomarkers to humans.

\section{miRNA biogenesis, turnover \& carriers in body fluids}

Most miRNAs are transcribed by RNA polymerase II into primary miRNAs (pri-miRNAs), which are produced in a wide array of lengths, often several thousand nucleotides long, and as seen with mRNA, are capped at the $5^{\prime}$ end and polyadenylated at the $3^{\prime}$ end. The transformation from pri-miRNAs to mature miRNA involves multiple steps at two cellular locations: in the nucleus, the pri-miRNAs are processed by the ribonuclease III enzyme Drosha in cooperation with its RNA binding partner Pasha (also called DGCR8) to stemloop-structured molecules called precursor miRNAs (pre-miRNAs); after exported into the cytoplasm, the pre-miRNAs are processed into approximately 22-nucleotide long mature RNA duplexes by the enzyme Dicer in cooperation with the another RNAbinding cofactor TRBP (also called loquacious, Loqs). After the cleavage, the mature miRNA duplex becomes a guide strand and a passenger strand. Although either strand of the duplex may potentially function as a mature miRNA, only one strand is incorporated into the RNA-induced silencing complex (RISC) [17]. Argonaute proteins, directly interacting with miRNAs, are key factors in the assembly and function of RISC [18].

Generally, most miRNA genes produce one dominant strand, while the opposite (star or passenger) strand is preferentially destroyed according to the 'use it or lose it' strategy. Through a large-scale study with eight in vitro cell lines, it was recently discovered that most miRNA have slow turnover rates, with no or minor decrease within $24 \mathrm{~h}$ [19]. While most detected miRNAs remain at $75 \%$ levels at $12 \mathrm{~h}$ after transcription inhibition, a small subset of miRNAs, mostly miRNA star strands turnover more quickly. For example, miR-21-3p (or miR-21*) had a fast turnover phase 
with half-life close to $1 \mathrm{~h}$. Cellular miRNA expression can be regulated by changes in transcriptional level, but post transcriptional modifications affect miRNA stability; therefore, the turnover rates of different miRNA isoforms are quite different. Using deep sequencing approaches, diverse nucleotide substitutions, additions and deletions have been detected in animal miRNAs. Interestingly, the addition of a single adenine to the $3^{\prime}$ end of miR-122 stabilizes the molecule [20]. Therefore, the extracellular miRNAs detected in body fluids may have diverse turnover rates.

In addition to the cellular miRNA function as a gene modulator, large numbers of miRNAs have been observed in the extracellular spaces of both healthy and diseased patients. So far, miRNA have been detected in a wide range of body fluids, including serum, plasma, urine, cerebrospinal fluid, saliva, breast milk and tears. It is known that circulating cell-free miRNAs are protected from nucleases in extracellular environments; however, synthetic miRNAs were rapidly degraded when combined with nondenatured plasma [5]. This suggests that extracellular miRNAs in body fluids are not stabilized by intrinsic chemical characteristics but by various types of carriers, such as a RNA binding protein complex or extracellular lipid vesicles. Extracellular vesicles are constantly released by almost all types of cells and are present in many body fluids such as blood, saliva and urine [21]. Based on their size, these extracellular lipid vesicles have been classified as exosomes $(\sim 100 \mathrm{~nm}$ in diameter) or microvesicles (1-10 $\mu \mathrm{m}$ in diameter). The constituents of lipid vesicles include proteins, DNA, mRNA, miRNA and lipids. When cells undergo stress, the extracellular lipid vesicles and their constituents change accordingly [22]. Using differential fraction approaches including ultracentrifuge, size-exclusion chromatography, filtration and immunoprecipitation, it was observed that a substantial amount of vesicle-free miRNAs were associated with Ago2 [23], which protects them from degradation. Recent studies indicate that miRNAs are actively or passively released from cells via several different processes. It is hypothesized that under normal conditions, the active release of miRNAs occurs in a regulated manner. In contrast, miRNAs may be released into the circulating system in a passive process during necrotic and/or apoptotic cell death [24]. It has been shown that miR-122 is able to shift between extracellular compartments under specific pathological conditions: increased with exosome-rich fraction in alcoholic liver disease and inflammation and as a protein-rich fraction in acetaminophen-induced liver necrosis [25]. In summary, the extracellular miRNAs detected in body fluids may have diverse turnover rates, affected by their sequence features and carriers.

\section{Analytical platforms for miRNA quantifications}

Extracellular miRNA abundance in body fluids can be measured by a number of platforms, such as quantitative real-time polymerase chain reaction ( $\mathrm{PPCR}$ ) and next-generation sequencing (NGS). These technologies have significantly contributed to the identification of new miRNAs and miRNA profiles in diseases have increased our understanding of miRNA biological functions. The isoforms of some miRNAs belong to the same family and may differ from each other by only one nucleotide, making selective measurement of each isoform difficult. NGS provides a high-throughput detection of novel miRNAs and high accuracy in distinguishing miRNA isomers. The qPCR platform provides a larger dynamic range of miRNA detection and, therefore, requires less sample volume and provides more sensitive measurements than NGS. Quantifying miRNA by qPCR can be accomplished by two common methods: relative quantification and absolute quantification. The former is based on internal controls to determine fold changes and the absolute quantification gives the exact copy number by comparison with a calibration curve. Detailed discussions of the strengths and pitfalls can be found in a previous review [26].

A variety of new assay methods have been developed and some are commercially available to measure miRNAs in body fluids. Droplet digital PCR (ddPCR) is a high-throughput system that permits PCR reactions in surfactant-stabilized droplets rather than 96-well plates [27]. It combines the nanoliter-sized droplet technology with digital PCR and enables processing of approximately 2 million reactions using conventional quantification assays. Compared with qPCR, ddPCR is able to obtain highly precise absolute quantification without external references, more tolerant to the presence of inhibitors of the amplification reaction and has greater precision and improved day-to-day reproducibility [28,29]. One recent article demonstrates the capability of using ddPCR to quantify the circulating miRNAs in serum and plasma [30]. Other innovative technologies of miRNA quantifications are high-resolution MS [31] and miRNA-derived fragment length polymorphism assay [32].

The small size of miRNAs, high similarity between miRNA family members and the low abundance of miRNAs in body fluids could lead to unacceptable false-positive and false-negative results in the identification of dysregulated miRNAs. Concerns have been raised about the comparability and accuracy of results from different assay methods. Recent analyses have revealed that the performance of these methods varies, with each platform having strengths and weaknesses. There is a critical need for standard approaches to mea- 
sure, report and compare the technical performance of different miRNA quantification platforms. To avoid interassay variance, it will be important to control preand postanalytical variables, such as RNA isolation methods, RNA quantification protocols, threshold Cq reporting and data normalization methods. In order to achieve accurate and reproducible measurements across different assays, it will be necessary to develop a set of synthetic small RNA sequences to be used as spike-in controls. The recently published microRNA quality control (miRQC) study compared and evaluated the performance of several miRNA quantification platforms [33]. Even the platforms based on the same technology may have substantial differences in reproducibility and specificity when evaluating miRNA expression. For low-input RNA, qPCR-based platforms seem to have a better score in sensitivity accompanied by high accuracy.

\section{Extracellular miRNAs as biomarkers of drug- induced cardiotoxicity}

Drug-induced cardiotoxicity is a major problem that has accounted for the failure of drug products during preclinical or clinical phases [13]. Many cancer therapy drugs are associated with a high risk of developing cardiotoxicity. For example, doxorubicin is an effective antineoplastic drug for many cancers; however, it also shows dose-dependent cardiotoxicity in both adult and pediatric patients. In addition to anthracyclines whose efficacy is jeopardized by cumulative cardiotoxicity, toxoids (e.g., taxol), alkylating agents (e.g., cyclophosphamide) and tyrosine kinase inhibitors (e.g., sunitinib) have been associated with cardiac dysfunction. In addition to the time- and money-consuming cardiac imaging techniques to monitor heart injury, serum protein biomarkers such as phosphokinase (CK), lactate dehydrogenase (LDH) and aspartate aminotransferase (AST) have been used to monitor heart injury. Recently, myosin light chain I (MLC-I), heart fatty acid binding protein (H-FABP), creatine kinase isoenzyme MB (CK-MB), B-type natriuretic peptide (BNP) and cardiac troponins [34] have been explored and used as biomarkers of myocardial cell injury [35]. BNP is a cardiac neurohormone released from heart ventricles in response to volume expansion and blood pressure changes [36]. Cardiac troponins I (cTnI) and T (cTnT) are part of the troponin complex and regulate cardiac muscle contraction. The utility of these proteins as clinical biomarkers of drug-induced cardiac toxicity is in progress and needs further study.

Drug-induced cardiotoxicity can be classified into two categories: structural damage with direct or indirect cellular injury, leading to ischemia; and functional damage due to cardiac ion channel interruption, lead- ing to arrhythmia. Structural toxicity may cause the release of some cellular molecules before loss of membrane integrity and investigators have studied the release of cardiac-specific miRNAs for potential use as circulating biomarkers in animals and human. A few rodent studies have been conducted to look at extracellular miRNA expression patterns during drug-induced cardiotoxicity. In 2009, the first study reported significant increases of plasma miR-208 as potential heartspecific biomarkers to detect isoproterenol-induced myocardial injury in rats [37]. Liu et al. investigated the potential of miR-208a as a noninvasive biomarker for myocardial injury and it appeared to be better than cTnI in a study that looked at $24 \mathrm{~h}$ treatment with isoproterenol in Sod2 $2^{+-}$and C57BL/6J wild-type mice [38]. A recent study explored circulating miRNA patterns in isoproterenol or doxorubicin treated rats; plasma miR-208 seems to be a sensitive biomarker for cardiotoxicity [39].

The following discussion will detail how heart-specific or muscle-specific miRNAs are being explored as biomarkers in human cardiac diseases. As one of the early studies to detect acute myocardial infarction (AMI) [40], circulating miRNAs, especially the heartspecific ones, have been explored. All muscle-enriched miRNAs, miR-1, miR-133 and miR-499, were found to be present at higher levels in the plasma of AMI patients within $4 \mathrm{~h}$ after the onset of infarction. As one of the cardiac-specific miRNAs, plasma miR-208a levels were increased at an early time point $(1 \mathrm{~h})$, while it was undetected in plasma from healthy controls, patients with renal infarction or non-AMI patients with chest pain [41]. Therefore, miR-208a seemed to be an AMI biomarker with high sensitivity and specificity. Using a ligation-induced AMI model in mice [42], the potential diagnostic roles of miR-208a and miR499 have been positively confirmed. In mice, it was observed that tissue miR-499 decreased in the myocardial infarct zone compared with the remote zone or the sham group. However, the D'Alessandra et al. [40]. AMI study failed to detect elevated miR-208a, and this may be explained by the relatively short half-life of miR-208a (3-12 h after AMI induction) in the blood. A family member, miR-208b, has been investigated and demonstrated to have a 1600 -fold increase in plasma of AMI patients [43]. In a recent study comparing heart- and muscle-specific circulating miRNAs with cTnI, all increased in a similar trend in advanced heart failure. With the support from a left ventricular assist device, the elevation of miRNAs reversed and dropped to detection limit within 3 months [44].

As a broadly muscle-specific (enriched in muscle, not selective in cardiac tissue) miRNA, miR-1 was detected with a 200 -fold peak elevation at $6 \mathrm{~h}$ of onset 
and stable levels for at least $24 \mathrm{~h}$ in AMI [45]. Ai and colleagues provide results supporting the use of circulating miR-1 as a biomarker for AMI and the increases seen in AMI patients appeared to be abrogated following medication [46]. miR-133 is another broadly muscle-specific miRNA that may serve to diagnose AMI. However, due to their expression in skeletal muscle, both miR-133 and miR-1 have been linked to other diseases and thus may not offer the desired specificity $[47,48]$.

Plasma levels of miR-423-5p have been found to be a strong diagnostic biomarker for heart failure (HF) patients, and distinguished patients with HF from healthy controls (AUC 0.91) and from patients with non-HF dyspnea (AUC 0.83) [49]. In an independent study, the elevation of circulating miR-423-5p was confirmed from HF patients relative to an age-, genderand ethnicity-matched control group [50]. In addition, a strong correlation $(\mathrm{p}=0.002)$ was observed between BNP and miRNA-score based on a panel of four miRNAs (miR-423-5p, miR-320a, miR-22 and miR92b). It is likely that the elevation of circulating miR$423-5 p$ in HF patients is caused by its upregulation in the human failing myocardium.

In conclusion, several studies fuel the notion that circulating miRNAs may serve as sensitive and specific biomarkers for heart injury. The findings from the studies described above clearly show the potential that miR-1, miR-133a, miR-499, miR-208 and miR423-5p hold as cardiac injury biomarkers in cardiovascular diseases. The combination of miRNAs with cTnI might be useful as diagnostic and prognostic tools for patients.

\section{Extracellular miRNA as biomarkers of drug- induced hepatotoxicity}

The liver is the main organ for the metabolism and detoxification of drugs and, therefore, is often exposed to the highest concentrations of orally consumed drugs [51]. Drug-induced liver injury (DILI) is one of the leading causes of drug attrition during development and post-marketing drug withdrawal [52]. DILI is a common adverse drug reaction seen in the clinic and can be classified into two subcategories: nonidiosyncratic (predictable) or idiosyncratic. A typical example of the former is acetaminophen (APAP or paracetamol) [53], and it occurs in a dose-dependent manner. Following a toxic dose of APAP, the conjugation pathways of the liver are overwhelmed and an increased proportion of APAP is metabolized to the high reactive metabolite $\mathrm{N}$-acetyl-p-benzoquinone imine. N-acetyl-p-benzoquinone imine can bind to cysteine residues in proteins, hereafter referred as APAP protein adducts. In contrast, idiosyncratic
DILI is unpredictable and can occur at a therapeutic dose. Currently, the clinically used DILI biomarkers are limited and imperfect. For example, serum levels of alanine aminotransferase (ALT) is the most commonly used biomarker of hepatocellular injury, but its elevation is not liver specific [54]. Consequently, it is not surprising that efforts continue to explore sensitive biomarkers as tools to provide diagnostic value and detect safety liabilities reliably during drug development.

Some liver-enriched miRNAs were found to be good candidates for new DILI biomarkers [55]. As one of the early studies to explore circulating miRNAs as liver injury biomarkers, Wang et al. [56] reported plasma increases in miRNA-122 and miRNA-192 with concurrent decreases in the liver from APAP-treated mice. In addition, the level of many plasma miRNAs inversely correlated with the level of hepatic miRNAs, suggesting that for these miRNAs, hepatic injury caused the release of the miRNAs into the circulation. Interestingly, the increases in both miR-122 and miR192 were detected earlier than the increase of ALT $(1 \mathrm{~h}$ post-treatment vs $3 \mathrm{~h}$ ) in the mice. In another APAP overdosed mice study, Bala et al. confirmed the significant increase of miR-122 in plasma [25]. In addition, both plasma miR-122 and ALT started to increase 3 and $6 \mathrm{~h}$ after APAP overdose, respectively, with a larger fold change increase in miR-122 than ALT. The robust increase in miR-122 was strongly associated with ALT elevation.

Similarly, the significant increase of miR-122 has been reported in APAP-overdosed rats [57-59], with increased serum levels of miR-122, miR-192 and miR193 at $3 \mathrm{~h}$ while ALT remained at baseline at this time period. At later time points, ALT and miR-122 were both increased and showed peak elevations between 12 and $24 \mathrm{~h}$ after APAP overdose. They returned to baseline at 3 days post-treatment in a similar pattern.

In addition to liver specific miRNAs, other types of miRNAs and their exported patterns have been investigated as liver injury biomarkers. In 2012, mouse models were used to study, APAP-induced liver injury, alcoholic liver disease and Toll-like receptor (TLR) 9+4 ligand-induced inflammatory cell-mediated liver damage. In addition to the robust increase of hepatospecific miR-122, immune-related miRNAs (miR-155, miR$146 \mathrm{a}$ and miR-125b) were identified and demonstrated mild increases in APAP-treated mice $(500 \mathrm{mg} / \mathrm{kg})$ [25]. The increase of these miRNAs could be the result of cell death (hepatocytes and immune cells). It is found that miR-122 and miR-155 were predominately associated with the protein fraction $6 \mathrm{~h}$ post dosing in the APAP model, whereas they were associated with the exosome-rich fraction in alcoholic liver disease induced in mice. 
To further evaluate the use of miR-122 as a clinically relevant marker of APAP-induced hepatotoxicity, several studies have been initiated to measure circulating miRNA levels in human patients $[25,60]$. In one clinical study, the potential of miR-122 and -192 as DILI biomarkers was explored in APAP-induced acute liver injury patients $(\mathrm{n}=53)$. It is reported that both serum miRNAs were elevated in APAP overdose patients compared with healthy controls. When each miRNA was compared against peak serum ALT, serum miR122 showed some association with ALT levels in the APAP acute liver injury cohort, whereas miR-192 showed no correlation. Another interesting observation is that miR-122 returned to baseline quicker than ALT [60]. In addition, the increase of miR-122 at earlier time points than ALT was upheld in another human DILI cohort [61]. A recent study looked at the utility of several mechanistic biomarkers in APAP overdose patients and reported that the initial measurements of miR-122, but not ALT, at first hospital presentation significantly correlated with peak ALT during the hospital stay [62]. Among patients whose initial ALT levels were in the normal range, miR-122 increases were higher in the patients who developed liver injury later, suggesting the elevation of miR-122 taken upon first presentation at the hospital could improve patient stratification and inform clinical care.

Importantly, circulating miRNA profiles could be used as biomarkers for APAP overdose diagnosis and discrimination from ischemic hepatitis [12]. Several circulating miRNAs (miR-122-5p, -27b-3p,-21-5p, $-125 b-5 p,-194-5 p,-193 a-5 p$ and -1290) contributed to distinguish APAP overdose from another common hepatotoxic pathology, ischemic hepatitis. This study also provided data on the evolution of miRNAs during treatment with the APAP antidote, $\mathrm{N}$-acetyl cysteine (NAC). A majority of the elevated miRNAs recovered quickly, but miR-1290 remained elevated for at least 2 days. In a small subset of patients with high peak levels of ALT, their miRNA profiles showed a rebound trend after stopping NAC treatment. Using the next-generation sequencing approach, a recent study [63] identified 36 miRNAs on a small number of samples $(\mathrm{n}=$ 6) from APAP-overdosed patients. Among them, eight miRNAs (miR-107, miR-122, miR-130a, miR-148a, miR-192, miR-22, miR-27b and miR-30a) overlapped with a previous APAP mice study [56]. Diverse isomiRs could perform differently in response to liver injury. Using a sequencing approach, it was identified that miR-23a with a $3^{\prime}$ end variation was increased after APAP overdose, while other forms showed no significant changes. Evaluating these miRNAs', some are enriched in liver: hsa-miR-122, hsa-miR-192, hsamiR-483-5p, hsa-miR-194-5p and hsa-miR-210-3p.
Seven APAP overdose-responsive miRNAs are highly expressed in tissues other than liver, such as muscle and pancreas. It is possible that these miRNAs are released from hepatocytes as they interact with key molecules in cell death for all tissues, or APAP overdose could cause multi-organ failure.

In our recent study [64] using a small RNA sequencing approach, eight serum miRNAs (miR-122, -375 , $-423-5$ p, -30d-5p, -125b-5p, -4732-5p, -204-5p and $-574-3 p)$ were identified with more than a twofold increase in APAP overdose pediatric patients $(n=8)$. Importantly, there was a strong correlation between serum miR-122 and APAP protein adducts $(\mathrm{p}<0.01)$. Sequential measurements at different days were made, and the time-dependent change of serum miR-122 followed ALT elevation. In one APAP overdose patient, the elevated serum miR-122 levels returned to baseline before serum ALT.

Compared with blood, urinary biomarkers are less invasive and may prove to be more amenable for kinetic studies. In 2012, urinary miRNA profiles were assessed for APAP-treated and carbon tetrachlorideinduced liver injury in rat models [65]. After administration of a toxic dose of APAP, 44 urinary miRNAs profiles were altered. The levels of ten common urinary miRNAs, miR-185, miR-296, miR-20b-3p, miR-484, miR-330*, miR-434, miR-433, miR-34C*, miR-291a-5p and miR-664, were increased in hepatotoxicant-treated rats. The nonhepatotoxicant, penicillin, did not induce any significant changes in these ten urinary miRNAs. To explore the translational nature of these urinary miRNAs, a recent clinical study was conducted in APAP overdose pediatric patients. Urine levels of miR-375, miR-940, miR-9-3p and miR-302a were demonstrated to be increased. In contrast to published results of blood borne miR-122, the urinary level of this biomarker was not significantly changed in APAP overdosed rat or human samples.

In summary, the extent of blood borne miR-122 increase may reflect the severity of liver injury. When compared with ALT, circulating miR-122 appears to be raised earlier, with a shorter half time of several hours. Many studies have focused on APAP as an experimental model, and future work should include additional DILI scenarios to explore their application. A group of miRNAs, rather than a single miRNA, may serve as specific and sensitive markers of liver injury.

\section{Extracellular miRNA as biomarkers of drug- induced nephrotoxicity}

Sensitive and specific biomarkers are needed to detect nephrotoxicity at earlier stages than is possible today. The kidney plays a crucial role in drug excretion and detoxification. Routinely used biomarkers are serum 
creatinine and blood urea nitrogen (BUN), both assessing the functional status of the kidney and detecting specific damage. Thus, these biomarkers increase significantly only after substantial kidney injury occurs and the damage may be irreversible. This stymies drug development because of the fear of inducing such morbidity as well as the clinical management of patients. Significant progress has been seen in identifying protein biomarkers of renal damage including $\beta-2$-microglobulin, clusterin, cystatinC, kidney injury molecule-1, albumin, urinary total protein and trefoil factor 3 in rodents [66,67]. Work is underway to see if these biomarkers can be translated to the clinic and prove useful in clinical trials and standard patient care.

Urine has been used for biomarker discovery of renal injury. It is noninvasive, directly associated with renal parenchymal changes and less complex than serum. For nonclinical assessment, many new potential miRNA biomarkers of nephrotoxicity have been identified using rodent models and compared with the traditional parameters. One translational study conducted at Vishal Vaidya's lab characterizes the miRNAs in rats with kidney injury (either induced by ischemia or gentamicin treatment) and patients with clinical diagnosis of acute kidney injury (AKI) [68]. A significant upregulation of tissue miR-21, -155 and -18a was observed in the animal models and the urinary miR-21 (AUC: 0.71; $\mathrm{p}=0.01$ ) and miR-155 (AUC: $0.7 ; \mathrm{p}=0.02$ ) profiles could distinguish AKI patients from controls. As a follow-up study, miR-155 deficient mice were generated and found to be more susceptible to renal injury induced by cisplatin than wild type mice. Based on gene expression analysis, c-Fos was identified as the target of miR-155 and increased apoptotic signals were found in the knockout mice [69]. Using two miRNA profiling platforms, qRT-PCR and small RNA-seq, urinary let-7d (decrease 177.8fold; $\mathrm{p}=0.0084$ ), miR-203 (decrease 5.428-fold; $\mathrm{p}=$ 0.0158 ) and miR-320 (increase 2.74-fold; $\mathrm{p}=0.0394$ ) were identified as potential biomarkers in gentamicintreated rats [70].

A collaborative program was performed under the Health and Environmental Sciences Institute (HESI) Biomarkers of Nephrotoxicity Committee and interesting observations were published as a companion paper in 2014 [71,72]. Cisplatin is a highly effective chemotherapeutic agent; however, the dose-dependent proximal tubule nephrotoxicity limits the achievable dosage and treatment intervals. Both studies employed a cisplatin-induced nephrotoxicity rat model to identify urine miRNA biomarkers. However, the study design differed in terms of dose, animal strain and feeding status. In one study [71], male Wistar rats received a single dose of cisplatin $(0,1$ and $3 \mathrm{mg} /$ $\mathrm{kg}$ ) and urinary miRNAs were analyzed at multiple days post treatment $(3,5,8,15$ and 26 days). Compared with vehicle controls at each time point, a large number of miRNAs reached a maximal increase on day 5 for $3 \mathrm{mg} / \mathrm{kg}$ treatment and then decreased to baseline levels on day 26. However, in this dose group, maximal tubular necrosis was detected on day 8 and the tubular regeneration was still seen on day 26. In another study, male Sprague-Dawley (SD) rats were treated with $1,3,6 \mathrm{mg} / \mathrm{kg}$ cisplatin, and 25 miRNAs were increased in urine [72]. Based on the histopathology, serum BUN, serum creatinine, urine Kim-1 and urine clusterin data, there is no significant difference between fed and fast condition. Similar to the previous observation, urine miR-let-7g-5p, miR-93-5p, miR191-5p and miR-192-5p showed an increasing trend on day 3 , maximizing levels on day 5 and dropping to control levels on day 7.

In 2011, Lorenzen et al. characterized plasma miRNA patterns among critically ill AKI patients (n $=77)$, healthy controls $(n=30)$ and acute myocardial infarction patients $(n=18)$. Circulating miR-210 was significantly increased in patients with AKI and the multivariable analysis indicated that miR-210 was predictive of 1-month mortality [73]. From another clinical evaluation of urinary miRNAs from AKI $(\mathrm{n}=98)$ and non-AKI ( $\mathrm{n}=97)$ patients, Ramachandran and colleagues reported a panel of four miRNAs (with higher miR-21, miR-200c, miR-423 and lower miR-4640) with a combined cross-validated AUC $(0.91 ; 95 \% \mathrm{CI}$ : 0.86-0.92) [74]. Normalization has been one of the biggest challenges in analyzing urinary miRNA data. This study reported three potential normalizers, miR307, miR-1287 and miR-489, with miR-1287 showing the most consistent performance (mean intraplate CV: $2.5 \%$; mean interplate CV: $3.5 \%)$.

In conclusion, several urinary miRNAs are reported to show aberrant levels in AKI patients, of which miR21 was shared between the studies. Large-scale studies are required to evaluate value as diagnostic biomarkers in AKI.

\section{Future perspective}

From the stand point of drug-induced toxicity, miRNAs have the potential to provide broad utility at various points in the drug development process. Extracellular miRNAs are great candidates as sensitive and specific biomarkers, and we can envision cell-free miRNA applications ranging all the way from nonclinical safety screening and clinical trial management to postmarket surveillance studies. There have been major advances in the discovery of miRNA species as tissue-specific toxicity biomarkers in a variety of 


\begin{tabular}{|c|c|c|c|c|c|}
\hline \multirow[t]{4}{*}{ Heart } & 17 control; $33 \mathrm{MI}$ & $\begin{array}{l}\text { miR-1, miR-133a, miR-133b, and } \\
\text { miR-499 }\end{array}$ & Plasma & $\operatorname{miR}-17-5 p$ & [40] \\
\hline & $\begin{array}{l}30 \text { control; } 33 \mathrm{MI}, 16 \text { non-MI } \\
C A D, 17 \text { other } C A D\end{array}$ & $\begin{array}{l}\text { miR-208a, miR-1, miR-133a, } \\
\text { and miR-499 }\end{array}$ & Plasma & cel-miR-39 spike-in & [41] \\
\hline & 39 controls; $20 \mathrm{HF}$ & $\operatorname{miR}-423-5 p$ & Plasma & $\operatorname{miR}-1249$ & [49] \\
\hline & 30 controls; $30 \mathrm{HF}$ & $\begin{array}{l}\text { miR-423-5p, mR-320a, miR-22, } \\
\text { miR-92b }\end{array}$ & Serum & Average $\mathrm{Ct}$ value & [50] \\
\hline \multirow[t]{3}{*}{ Liver } & 25 controls; 53 APAP & miR-122, miR-192 & Plasma & U6 snRNA & [60] \\
\hline & 129 APAP & $\operatorname{miR}-122$ & Plasma & let-7d & [62] \\
\hline & $\begin{array}{l}10 \text { controls; } 10 \text { therapeutic } \\
\text { APAP, } 8 \text { APAP overdose }\end{array}$ & miR-122 (Serum), miR-375 & Serum/urine & $\begin{array}{l}\text { let-7d (serum), miR- } \\
671-3 p \text { (urine) }\end{array}$ & [64] \\
\hline \multirow[t]{3}{*}{ Kidney } & 30 controls; $77 \mathrm{AKI} ; 18 \mathrm{MI}$ & miR-210, miR-16, miR-320 & Plasma & cel-miR-54 spike-in & [73] \\
\hline & 25 controls; 22 AKI & miR-21, miR-155 & Urine & Equal amounts of RNA & [68] \\
\hline & 97 non $\mathrm{AKI} ; 98 \mathrm{AKI}$ & $\begin{array}{l}\text { miR-21, miR-200c, miR-423, } \\
\text { miR-4640 }\end{array}$ & Urine & miR-1287 & [74] \\
\hline
\end{tabular}

clinical settings; however, some miRNAs' lack of tissue specificity raises questions as to their usefulness as specific biomarkers. In the next decade, we still need a better understanding of the biological and analytic factors that affect the quantification of extracellular miRNAs.

Currently, the development of miRNAs as clinical biomarkers has been hindered by lack of standardization and concerns about the comparability of results from different assay methods. Therefore, extracellular miRNA-based biomarkers have not yet been used as diagnostic tools in clinical applications. We expect that future work that examines the accuracy, specificity, reproducibility and robustness of these miRNAs in large multicenter clinical studies will be helpful in determining their value as biomarkers. At the same time, the ongoing process of new miRNA discovery and development of quantification methods will help us enhance our ability to improve the prediction and detection of drug induced toxicities.

We expect to see further examination of these miRNA biomarker candidates in future studies conducted with more optimized study designs. First, more frequent sampling in the early stages of injury may generate more timely data that can compare the kinetic profiles of candidate biomarkers to one another.
Second, it will be recommended to match the miRNA samples to ones that provide concurrent measurement of traditional blood chemistry data as well. Finally, the inclusion of another type of organ damage control to develop tissue-specific miRNA biomarkers should be included in future study design. Whether extracellular miRNAs are better predictive molecules than existing clinical biomarkers awaits confirmation in future clinical studies (Table 1).

\section{Disclaimer}

This article is not an official guidance or policy statement of the US Food and Drug Administration (FDA). No official support or endorsement by the FDA is intended or should be inferred.

\section{Financial \& competing interests disclosure}

The authors have no relevant affiliations or financial involvement with any organization or entity with a financial interest in or financial conflict with the subject matter or materials discussed in the manuscript. This includes employment, consultancies, honoraria, stock ownership or options, expert testimony, grants or patents received or pending, or royalties.

No writing assistance was utilized in the production of this manuscript. 
Executive summary

miRNA background

- MicroRNAs (miRNAs) are small noncoding RNAs that post-transcriptionally regulate the expression of thousands of genes in both normal and disease contexts.

miRNA biogenesis, turnover \& carriers in body fluids

- Extracellular miRNAs are emerging as noninvasive biomarkers for drug-induced toxicities because they offer many attractive features: stability, tissue-specificity and evolutionarily conserved sequences.

Analytical platforms for miRNA quantifications

- Technological advances have spawned a multitude of platforms for miRNA profiling, and most common techniques for miRNA quantification are based on QPCR, hybridization and next-generation sequencing.

- A variety of new assay methods have been developed to measure miRNAs in body fluids, such as droplet digital PCR (ddPCR). Currently, qPCR based platforms seem to have a better sensitivity accompanied by high accuracy.

Extracellular miRNAs as biomarkers of drug-induced cardiotoxicity

- Circulating miR-1, miR-133a, miR-499, miR-208 and miR-423-5p are promising cardiac injury biomarkers in cardiovascular diseases.

Extracellular miRNAs as biomarkers of drug-induced hepatotoxicity

- Liver-enriched miR-122 has been identified as an APAP injury biomarker in animal and clinical studies.

Extracellular miRNAs as biomarkers of drug-induced nephrotoxicity

- Several urinary miRNAs are reported to show aberrant levels in kidney injury patients, of which miR-21 was shared between the studies.

Future perspective

- Validation of candidate miRNAs in larger study cohorts and accurate measurement of low amounts of miRNA in body fluids are the main challenges for their use as clinical biomarkers.

\section{References}

Papers of special note have been highlighted as: $\bullet$ of interest

1 Friedman RC, Farh KK-H, Burge CB, Bartel DP. Most mammalian mRNAs are conserved targets of microRNAs. Genome Res. 19(1), 92-105 (2009).

2 Bazzini AA, Lee MT, Giraldez AJ. Ribosome profiling shows that mir- 430 reduces translation before causing mRNA decay in zebrafish. Science 336(6078), 233-237 (2012).

3 Kawasaki H, Taira K. MicroRNA-196 inhibits hoxb8 expression in myeloid differentiation of hl60 cells. Nucleic Acids Symp. Ser. (Oxf.) 48(1), 211-212 (2004).

4 Lee RC, Feinbaum RL, Ambros V. The c. Elegans heterochronic gene lin- 4 encodes small RNAs with antisense complementarity to lin-14. Cell 75(5), 843-854 (1993).

5 Mitchell PS, Parkin RK, Kroh EM et al. Circulating microRNAs as stable blood-based markers for cancer detection. Proc. Natl Acad. Sci. USA 105(30), 10513-10518 (2008).

6 Chen X, Ba Y, Ma L et al. Characterization of microRNAs in serum: a novel class of biomarkers for diagnosis of cancer and other diseases. Cell Res. 18(10), 997-1006 (2008).

7 miR2Disease Base. www.mir2disease.org/

8 Li Y, Qiu C, Tu J et al. Hmdd v2.0: A database for experimentally supported human microRNA and disease associations. Nucleic Acids Res. 42(D1), D1070-D1074 (2014).

9 Lagos-Quintana M, Rauhut R, Yalcin A, Meyer J, Lendeckel W, Tuschl T. Identification of tissue-specific microRNAs from mouse. Curr. Biol. 12(9), 735-739 (2002).

10 Liang Y, Ridzon D, Wong L, Chen C. Characterization of microRNA expression profiles in normal human tissues. BMC Genomics 8(1), 166 (2007).
11 Guo Z, Maki M, Ding R, Yang Y, Zhang B, Xiong L. Genome-wide survey of tissue-specific microRNA and transcription factor regulatory networks in 12 tissues. Sci. Rep. 4, 5150 (2014).

12 Ward J, Kanchagar C, Veksler-Lublinsky I et al. Circulating microRNA profiles in human patients with acetaminophen hepatotoxicity or ischemic hepatitis. Proc. Natl Acad. Sci. USA 111(33), 12169-12174 (2014).

13 Cook D, Brown D, Alexander R et al. Lessons learned from the fate of astrazeneca's drug pipeline: a five-dimensional framework. Nat. Rev. Drug Discov. 13(6), 419-431 (2014).

14 Cui Y, Paules RS. Use of transcriptomics in understanding mechanisms of drug-induced toxicity. Pharmacogenomics 11(4), 573-585 (2010).

15 US FDA. Guidance for industry pharmacogenomic data submissions (2006).

16 US FDA. Guidance for industry: qualification process for drug development tools. (2010).

17 Krol J, Loedige I, Filipowicz W. The widespread regulation of microRNA biogenesis, function and decay. Nat. Rev. Genet. 11(9), 597-610 (2010).

18 Chendrimada TP, Gregory RI, Kumaraswamy E et al. Trbp recruits the dicer complex to ago 2 for microRNA processing and gene silencing. Nature 436(7051), 740-744 (2005).

19 Guo Y, Liu J, Elfenbein SJ et al. Characterization of the mammalian miRNA turnover landscape. Nucleic Acids Res. (2015) (Epub ahead of print).

20 Katoh T, Sakaguchi Y, Miyauchi K et al. Selective stabilization of mammalian microRNAs by 3' adenylation mediated by the cytoplasmic poly(a) polymerase gld-2. Genes Dev. 23(4), 433-438 (2009). 
21 Witwer KW, Buzás EI, Bemis LT et al. Standardization of sample collection, isolation and analysis methods in extracellular vesicle research. J. Extracell. Vesicles 2, 10.3402 (2013).

22 Kucharzewska P, Christianson HC, Welch JE et al. Exosomes reflect the hypoxic status of glioma cells and mediate hypoxia-dependent activation of vascular cells during tumor development. Proc. Natl Acad. Sci. USA 110 (18), 7312-7317 (2013).

23 Arroyo JD, Chevillet JR, Kroh EM et al. Argonaute2 complexes carry a population of circulating microRNAs independent of vesicles in human plasma. Proc. Natl Acad. Sci. USA 108(12), 5003-5008 (2011).

- Interesting study exploring the miRNAs profiles in exosomes and Ago2 complexes.

24 Roderburg C, Luedde T. Circulating microRNAs as markers of liver inflammation, fibrosis and cancer. J Hepatol. 61(6), 1434-1437 (2014).

25 Bala S, Petrasek J, Mundkur S et al. Circulating microRNAs in exosomes indicate hepatocyte injury and inflammation in alcoholic, drug-induced, and inflammatory liver diseases. Hepatology 56(5), 1946-1957 (2012).

26 Pritchard CC, Cheng HH, Tewari M. MicroRNA profiling: Approaches and considerations. Nat. Rev. Genet. 13(5), 358-369 (2012).

27 Hindson BJ, Ness KD, Masquelier DA et al. Highthroughput droplet digital PCR system for absolute quantitation of DNA copy number. Anal. Chem. 83(22), 8604-8610 (2011).

28 Hindson CM, Chevillet JR, Briggs HA et al. Absolute quantification by droplet digital PCR versus analog real-time PCR. Nat. Meth. 10(10), 1003-1005 (2013).

29 Dingle TC, Sedlak RH, Cook L, Jerome KR. Tolerance of droplet-digital PCR vs real-time quantitative PCR to inhibitory substances. Clin. Chem. 59(11), 1670-1672 (2013).

30 Miotto E, Saccenti E, Lupini L, Callegari E, Negrini M, Ferracin M. Quantification of circulating miRNAs by droplet digital PCR: comparison of evagreen- and taqmanbased chemistries. Cancer Epidemiol. Biomarkers Prev. 23(12), 2638-2642 (2014).

31 Kullolli M, Knouf E, Arampatzidou M, Tewari M, Pitteri SJ. Intact microRNA analysis using high resolution mass spectrometry. J. Am. Soc. Mass Spectrom. 25(1), 80-87 (2014).

32 Xie X, Tang F, Yang Z et al. MicroRNA-derived fragment length polymorphism assay. Sci. Rep. 5, 9356 (2015).

33 Mestdagh P, Hartmann N, Baeriswyl L et al. Evaluation of quantitative miRNA expression platforms in the microRNA quality control (MIRQC) study. Nat. Meth. 11(8), 809-815 (2014).

- Recently published microRNA quality control (miRQC) study evaluating the performance of several miRNA quantification platforms.

34 Bertinchant JP, Polge A, Juan JM et al. Evaluation of cardiac troponin $i$ and $t$ levels as markers of myocardial damage in doxorubicin-induced cardiomyopathy rats, and their relationship with echocardiographic and histological findings. Clin. Chim. Acta 329(1-2), 39-51 (2003).

35 Kehl DW, Iqbal N, Fard A, Kipper BA, De La Parra Landa A, Maisel AS. Biomarkers in acute myocardial injury. Transl. Res. 159(4), 252-264 (2012).

36 Baughman KL. B-type natriuretic peptide - a window to the heart. N. Engl. J. Med. 347(3), 158-159 (2002).

37 Ji X, Takahashi R, Hiura Y, Hirokawa G, Fukushima Y, Iwai N. Plasma mir-208 as a biomarker of myocardial injury. Clin. Chem. 55(11), 1944-1949 (2009).

38 Liu L, Aguirre SA, Evering WEN et al. Mir-208a as a biomarker of isoproterenol-induced cardiac injury in $\mathrm{SOD}^{+/-}$and c57bl/6j wild-type mice. Toxicol. Pathol. 42(7), 1117-1129 (2014).

39 Nishimura Y, Kondo C, Morikawa Y et al. Plasma mir-208 as a useful biomarker for drug-induced cardiotoxicity in rats. J. Appl. Toxicol. 35(2), 173-180 (2015).

40 D'alessandra Y, Devanna P, Limana F et al. Circulating microRNAs are new and sensitive biomarkers of myocardial infarction. Eur. Heart J. 31(22), 2765-2773 (2010).

41 Wang G-K, Zhu J-Q, Zhang J-T et al. Circulating microRNA: a novel potential biomarker for early diagnosis of acute myocardial infarction in humans. Eur. Heart J. 31(6), 659-666 (2010).

- Interesting study showing that circulating miRNA could provide diagnostic value for cardiovascular disease.

42 Xiao J, Shen B, Li J et al. Serum microRNA-499 and microRNA-208a as biomarkers of acute myocardial infarction. Int. J. Clin. Exp. Med. 7(1), 136-141 (2014).

43 Corsten MF, Dennert R, Jochems S et al. Circulating microRNA-208b and microRNA-499 reflect myocardial damage in cardiovascular disease. Circ. Cardiovasc. Genet. 3(6), 499-506 (2010).

44 Akat KM, Moore-Mcgriff DV, Morozov P et al. Comparative RNA-sequencing analysis of myocardial and circulating small RNAs in human heart failure and their utility as biomarkers. Proc. Natl Acad. Sci. USA 111(30), 11151-11156 (2014).

45 Cheng Y, Tan N, Yang J et al. A translational study of circulating cell-free microRNA-1 in acute myocardial infarction. Clin. Sci. (Lond.) 119(2), 87-95 (2010).

46 Ai J, Zhang R, Li Y et al. Circulating microRNA-1 as a potential novel biomarker for acute myocardial infarction. Biochem. Biophys. Res. Commun. 391(1), 73-77 (2010).

$47 \mathrm{Ng}$ EK, Chong WW, Jin $\mathrm{H}$ et al. Differential expression of microRNAs in plasma of patients with colorectal cancer: a potential marker for colorectal cancer screening. Gut 58(10), 1375-1381 (2009).

48 Hu Z, Chen X, Zhao Y et al. Serum microRNA signatures identified in a genome-wide serum microRNA expression profiling predict survival of non-small-cell lung cancer. J. Clin. Oncol. 28(10), 1721-1726 (2010).

49 Tijsen AJ, Creemers EE, Moerland PD et al. Mir423-5p as a circulating biomarker for heart failure. Circ. Res. 106(6), 1035-1039 (2010).

- Interesting study showing that circulating miR-423-5p might be biomarker for heart failure. 
50 Goren Y, Kushnir M, Zafrir B, Tabak S, Lewis BS, Amir O. Serum levels of microRNAs in patients with heart failure. Eur. J. Heart Fail. 14(2), 147-154 (2012).

51 Wrighton SA, Stevens JC. The human hepatic cytochromes p450 involved in drug metabolism. Crit. Rev. Toxicol. 22(1), 1-21 (1992).

52 Lewis JH. The art and science of diagnosing and managing DILI in 2015 and beyond. Clin. Gastroenterol. Hepatol. (2015) (Epub ahead of print).

53 Beger R, Bhattacharyya S, Yang X et al. Translational biomarkers of acetaminophen-induced acute liver injury. Arch. Toxicol. 1-26 (2015).

54 Yang R-Z, Blaileanu G, Hansen BC, Shuldiner AR, Gong D-W. cDNA cloning, genomic structure, chromosomal mapping, and functional expression of a novel human alanine aminotransferase. Genomics 79(3), 445-450 (2002).

55 Laterza OF, Lim L, Garrett-Engele PW et al. Plasma microRNAs as sensitive and specific biomarkers of tissue injury. Clin. Chem. 55(11), 1977-1983 (2009).

- Leading study exploring plasma miR-122 as a circulating biomarkers for liver injury.

56 Wang K, Zhang S, Marzolf B et al. Circulating microRNAs, potential biomarkers for drug-induced liver injury. Proc. Natl Acad. Sci. USA 106(11), 4402-4407 (2009).

- Pioneering study showing that miRNAs might be used as noninvasive biomarkers for drug induced liver injury in animal model.

57 Starckx S, Batheja A, Verheyen GR et al. Evaluation of mir122 and other biomarkers in distinct acute liver injury in rats. Toxicol. Pathol. 41(5), 795-804, (2012).

58 Su YW, Chen X, Jiang ZZ et al. A panel of serum microRNAs as specific biomarkers for diagnosis of compound- and herb-induced liver injury in rats. PLoS ONE 7(5), e37395 (2012)

59 Yamaura Y, Nakajima M, Takagi S, Fukami T, Tsuneyama $\mathrm{K}$, Yokoi T. Plasma microRNA profiles in rat models of hepatocellular injury, cholestasis, and steatosis. PLoS ONE 7(2), e30250 (2012).

60 Starkey Lewis PJ, Dear J, Platt V et al. Circulating microRNAs as potential markers of human drug-induced liver injury. Hepatology 54(5), 1767-1776 (2011).

- First clinical study exploring liver-enriched miR-122 as hepatotoxicity biomarker in human.

61 Thulin P, Nordahl G, Gry M et al. Keratin-18 and microRNA-122 complement alanine aminotransferase as novel safety biomarkers for drug-induced liver injury in two human cohorts. Liver Int. 34(3), 367-378 (2013).

62 Antoine DJ, Dear JW, Lewis PS et al. Mechanistic biomarkers provide early and sensitive detection of acetaminophen-induced acute liver injury at first presentation to hospital. Hepatology 58(2), 777-787 (2013).

63 Krauskopf J, Caiment F, Claessen SM et al. Application of high-throughput sequencing to circulating microRNAs reveals novel biomarkers for drug-induced liver injury. Toxicol. Sci. 143(2), 268-276 (2015).

64 Yang X, Salminen WF, Shi Q et al. Potential of extracellular microRNAs as biomarkers of acetaminophen toxicity in children. Toxicol. Appl. Pharmacol. 284(2), 180-187 (2015).

65 Yang X, Greenhaw J, Shi Q et al. Identification of urinary microRNA profiles in rats that may diagnose hepatotoxicity. Toxicol. Sci. 125(2), 335-344 (2012).

66 US FDA. Review of Qualification Data for Biomarkers of Nephrotoxicity Submitted by the Predictive Safety Testing Consortium. www.fda.gov

67 EMEA/FDA guideline on detection of drug-induced nephrotoxicity (2009).

http://www.ema.europa.eu

68 Saikumar J, Hoffmann D, Kim T-M et al. Expression, circulation, and excretion profile of microRNA-21, -155, and -18a following acute kidney injury. Toxicol. Sci. 129(2), 256-267 (2012).

- Interesting study showing that miRNA profiles are useful biomarkers for kidney injury.

69 Pellegrini KL, Han T, Bijol V et al. MicroRNA-155 deficient mice experience heightened kidney toxicity when dosed with cisplatin. Toxicol. Sci. 141(2), 484-492 (2014).

70 Nassirpour R, Mathur S, Gosink M et al. Identification of tubular injury microRNA biomarkers in urine: Comparison of next-generation sequencing and qPCR-based profiling platforms. BMC Genomics 15(1), 485 (2014).

71 Pavkovic M, Riefke B, Ellinger-Ziegelbauer H. Urinary microRNA profiling for identification of biomarkers after cisplatin-induced kidney injury. Toxicology 324(0), 147-157 (2014).

72 Kanki M, Moriguchi A, Sasaki D et al. Identification of urinary miRNA biomarkers for detecting cisplatin-induced proximal tubular injury in rats. Toxicology $324(0), 158-168$ (2014).

73 Lorenzen JM, Kielstein JT, Hafer C et al. Circulating mir-210 predicts survival in critically ill patients with acute kidney injury. Clin. J. Am. Soc. Nephrol. 6(7), 1540-1546 (2011).

74 Ramachandran K, Saikumar J, Bijol V et al. Human mirnome profiling identifies microRNAs differentially present in the urine after kidney injury. Clin. Chem. 59(12), 1742-1752 (2013). 